OPEN ACCESS

Edited by:

John Doonan,

Aberystwyth University, UK

Reviewed by:

Henrik Buschmann

University of Osnabrück, Germany

Amy T. Hark,

Muhlenberg College, USA

*Correspondence:

Huazhong Ren

renhuazhong@cau.edu.cn

Specialty section:

This article was submitted to Crop Science and Horticulture,

a section of the journal

Frontiers in Plant Science

Received: 20 May 2016

Accepted: 22 July 2016

Published: 10 August 2016

Citation:

Liu X, Bartholomew E, Cai Y and Ren H (2016) Trichome-Related Mutants Provide a New Perspective on Multicellular Trichome Initiation and Development in Cucumber (Cucumis sativus L). Front. Plant Sci. 7:1187.

doi: $10.3389 /$ fpls. 2016.01187

\section{Trichome-Related Mutants Provide a New Perspective on Multicellular Trichome Initiation and Development in Cucumber (Cucumis sativus L)}

\author{
Xingwang Liu 1,2, Ezra Bartholomew ${ }^{1,2}$, Yanling Cai ${ }^{2}$ and Huazhong Ren ${ }^{1,2 *}$ \\ ${ }^{1}$ College of Horticulture, China Agricultural University, Beijing, China, ${ }^{2}$ Beijing Key Laboratory of Growth and Developmental \\ Regulation for Protected Vegetable Crops, China Agricultural University, Beijing, China
}

Trichomes are specialized epidermal cells located in aerial parts of plants that function in plant defense against biotic and abiotic stresses. The simple unicellular trichomes of Arabidopsis serve as an excellent model to study the molecular mechanism of cell differentiation and pattern formation in plants. Loss-of-function mutations in Arabidopsis thaliana have suggested that the core genes GL1 (which encodes a MYB transcription factor) and TTG1 (which encodes a WD40 repeat-containing protein) are important for the initiation and spacing of leaf trichomes, while for normal trichome initiation, the genes GL3, and EGL3 (which encode a bHLH protein) are needed. However, the positive regulatory genes involved in multicellular trichrome development in cucumber remain unclear. This review focuses on the phenotype of mutants (csg/3, tril, tbh, mict, and csg/1) with disturbed trichomes in cucumber and then infers which gene(s) play key roles in trichome initiation and development in those mutants. Evidence indicates that MICT, $T B H$, and CsGL1 are allelic with alternative splicing. CsGL3 and TRIL are allelic and override the effect of $T B H, M I C T$, and CSGL1 on the regulation of multicellular trichome development; and affect trichome initiation. CsGL3, TRIL, MICT, TBH, and CsGL1 encode HD-Zip proteins with different subfamilies. Genetic and molecular analyses have revealed that CSGL3, TRIL, MICT, TBH, and CSGL1 are responsible for the differentiation of epidermal cells and the development of trichomes. Based on current knowledge, a positive regulator pathway model for trichome development in cucumber was proposed and compared to a model in Arabidopsis. These data suggest that trichome development in cucumber may differ from that in Arabidopsis.

Keywords: unicellular, arabidopsis, multicellular, cucumber, mutants, trichome-related genes, regulator pathway

\section{INTRODUCTION}

Plant trichomes are highly specialized epidermal protrusions that are located on the surfaces of leaves, stems, petioles, sepals, seed coats, and other aerial organs. Their diversity is almost as great as the number of species on which they are found. Morphologically, they can be unicellular or multicellular as well as secretory glandular or non-glandular (Hülskamp et al., 1998; Hülskamp, 2004; Tissier, 2012; Chen et al., 2014). In the model plant Arabidopsis thaliana, 
extensive studies have been performed on unicellular trichome development, especially on leaves (Hülskamp, 2004; Ishida et al., 2008; Pesch and Hülskamp, 2009). Classical molecular genetic approaches have identified several regulators that work in distinct developmental processes, such as trichome initiation/formation, endo-reduplication, and branch construction, and growth orientation (Schwab et al., 2000; Szymanski et al., 2000; Chen et al., 2014). The regulatory pathways of unicellular trichome development comprise both positive (mutants develop fewer trichomes) and negative (mutants develop more and/or clusters of trichomes) transcription factors (Ishida et al., 2008; Balkunde et al., 2010; Grebe, 2012). The crucial positive transcription factors belong to three protein classes: one WD40-repeat protein TRANSPARENT TESTA GLABRA1 (TTG1) (Galway et al., 1994; Walker et al., 1999); four basic helix-loop-helix (bHLH) proteins: GLABRA3 (GL3)m ENHANCER OF GLABRA3 (EGL3) (Payne et al., 2000; Zhang et al., 2003), TRANSPARENT TESTA (TT8) (Zhang et al., 2003), and MYC-1 (Zhao et al., 2012); and three R2-R3 type-MYB transcription factors: GLABRA1 (GL1), MYB23 and MYB5 (Oppenheimer et al., 1991; Li et al., 2009; Tominaga-Wada et al., 2012). They bind together to form an MYB-bHLH-WD40 (MBW) trimeric complex that activates the downstream gene GL2 (GLABRA2), which initiates trichome differentiation (Pesch and Hülskamp, 2009). Moreover, several small, single-repeat MYB-negative regulatory proteins, such as TRIPTYCHON (TRY), CAPRICE (CPC), ENHANCER OF TRYAND CPC1 (ETC1), ECT2, ETC3, CAPRICE-LIKE MYB3, and TRICHOMELESS1, and TRICHOMELESS2 (TCL1, and TCL2, respectively), have been shown to act in a non-cellautonomous manner (Wada et al., 1997, 2002; Esch et al., 2004; Kirik et al, 2004; Pesch and Hülskamp, 2009; Wester et al., 2009; Edgar et al., 2014; Hauser, 2014; Wang and Chen, 2014). They compete with the R2R3 MYB protein GL1 and bind to bHLH proteins, including GL3/EGL3, to suppress trichome initiation in adjacent cells (Wang et al., 2014).

Cucumber (Cucumis sativus L.), as one of the most important vegetable crops, is also covered with trichomes ranging from the stems and leaves to the flowers, branches, fruits, and tendrils. During early fruit development, deep ridges along the length of the fruit cover the fruit surface, and densely spaced fruit trichomes are randomly scattered relative to the ridges (Liu et al., 2011; Chen et al., 2014). Trichomes on cucumber fruit are called spines. In cucumber, the fruit spine combines with tubercles to form the warty fruit trait, which is a very important fruit quality trait (Chen et al., 2014; Li et al., 2015). Compared to warty fruit, smooth fruit, with no spines, or tubercles, is very important for the breeding of freshly eaten cucumber types, as they are easier to clean, package, transport and store (Zhang et al., 2010; Yang et al., 2014). Moreover, smooth fruit is becoming increasingly popular due to its attractive

Abbreviations: TRIL, trichome-less; TBH, tiny branched hair; MICT, microtrichome; TTG1, transparent testa glabra1; bHLH, basic helix-loop-helix; GL3, glabra3; EGL3, enhancer of glabra3; MBW, MYB-bHLH-WD40; TRY, triptychon; CPC, caprice; ETC1, enhancer of tryand caprice1; HD-Zip, homeodomain-leucine zipper; PDF2, protodermal factor2; ATML1, Arabidopsis thaliana meristem layer1; MADS, $\underline{M} C M I-\underline{A} G A M O U S-\underline{D E F I C I E N S-S R F 4}$ and distinctly shiny appearance (Li et al., 2015; Pan et al., 2015; Cui et al., 2016). Despite the importance of fruit spines in cucumber breeding for external quality, there are limited reports of the regulation of fruit spine development and few detailed characterizations of cucumber genes with disturbed trichome development. To understand trichome development in cucumber, we must determine the crucial regulators for its initiation and formation based on cucumber trichome-related mutants.

We begin this review by focusing on cucumber trichomerelated mutants and the role that they play in trichome formation in plants with multicellular trichomes. Several mutants, such as trichome-less (tril) (Wang et al., 2016), glabrous 3 (csgl3) (Pan et al., 2015; Cui et al., 2016), tiny branched hair (tbh) (Chen et al., 2014), micro-trichome (mict) (Zhao et al., 2015a), and glabrous 1 (csgl1) (Li et al., 2015), inhibit trichome development via different mechanisms, but all of these mutants cause a reduction in one type of spine. The possible mechanisms involved in modulating trichome development in cucumber mutants are summarized herein.

\section{MUTANTS WITH DISTURBED TRICHOME IN CUCUMBER}

Five trichome-related mutants have been reported in cucumber (Chen et al., 2014; Li et al., 2015; Pan et al., 2015; Zhao et al., 2015a; Cui et al., 2016; Wang et al., 2016). Wild-type cucumber fruits have two types of trichomes, both of which are multicellular (Figures 1, 2) (Chen et al., 2014). Type I trichomes are small, glandular trichomes, with a 3- to 5-cell base topped with a 4 - to 8 -cell head. Type II trichomes, which predominate, are much larger, non-glandular trichomes that are composed of a base and stalk (Figure 2). Compared to wild-type cucumber plants, all mutants appeared to be glabrous with no noticeable trichomes on the leaves, stems, tendrils, floral organs, or fruits (Table 1, Figures 1, 2). Each mutant has a trichome type except for the mutants tril and csgl3 (Figure 2). Trichomes have been reclassified into three morphologies according to their shape on leaves and fruit surfaces. Type I trichomes, which are found in the $t b h$, mict, and csgll mutants, have a small papillar-shaped head (Figures 2A,D,F). Type II trichomes, which exist in the tbh and mict mutants, consist of one to five rounded cells without the pyramid-shaped head or pieshaped base (Figures 2B,E). However, type III trichomes occur only in tbh mutants; this type of trichome was not previously described. Here, we classified these trichomes into the new type III according to the branching at the top of the trichome (Figure 2C). Trichome phenotypes from tbh, mict and csgll indicated that these three mutants are all involved in trichome development and not in trichome initiation. Interestingly, the tril and csgl3 mutants showed a completely glabrous phenotype on the shoot and fruit epidermis (Figures 2G,H), suggesting that TRIL and CsGL3 may be upstream positive regulators of $T B H, M I C T$, and CsGL1 for the regulation of multicellular trichome development and may affect epidermal cell initiation. 

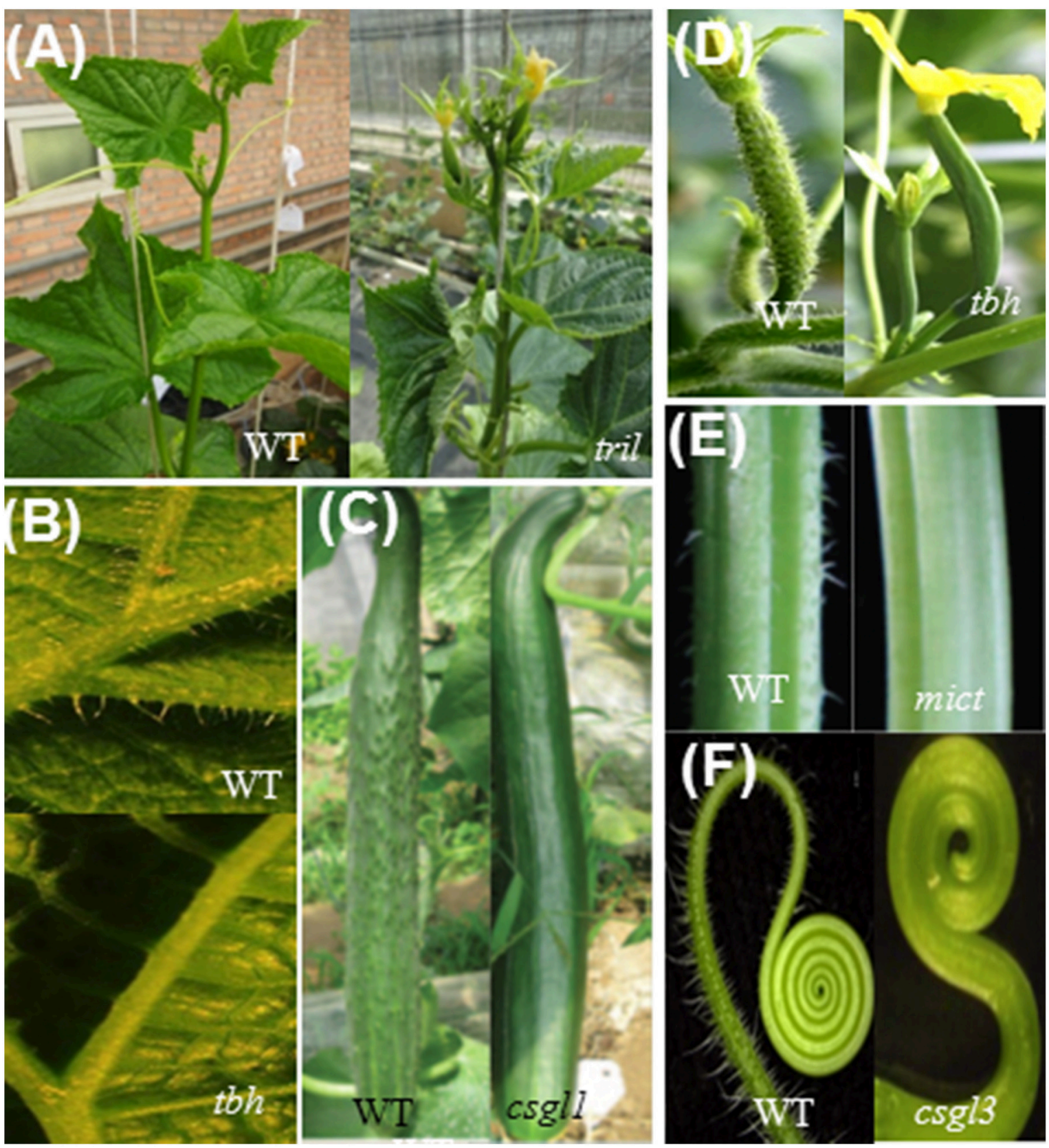

FIGURE 1 | Phenotypic characterization of mutants and the wild type (WT). (A) WT and tril plants; (B) Leaves; (C) Fruits; (D). Fruit 0 days after anthesis; (E) Stems; (F) Tendril. Picture (A) is from Wang et al. (2016); Pictures (B,D) are from Chen et al. (2014); and Pictures (C,E) are from Li et al. (2015); Picture (F) cited form Pan et al. (2015).

Compared to the multicellular trichomes found on aerial organs, such as leaves and fruits, the trichomes found on underground organs, such as root hairs, are characterized as single-celled, unbranched, elongated, and soft-structured with small tumors attached. Based on the published results, there was no difference between the wild-type and the tbh, mict, and csgll mutants (Chen et al., 2014; Li et al., 2015; Zhao et al., 2015a), indicating that the TBH, MICT, and CsGL1 mutants may not be involved in root hair formation. In contrast, the root length and number of branches of the tril mutant increased, suggesting that root hair formation in cucumber might be regulated by TRIL (Wang et al., 2016).

Other remarkable differences (except for the common phenotype) may exist among each mutant, such as dwarfism, branching, leaf curvature, and petal opening rates. In mict and $t b h$, rounded-head trichomes were found on the hypocotyls, 


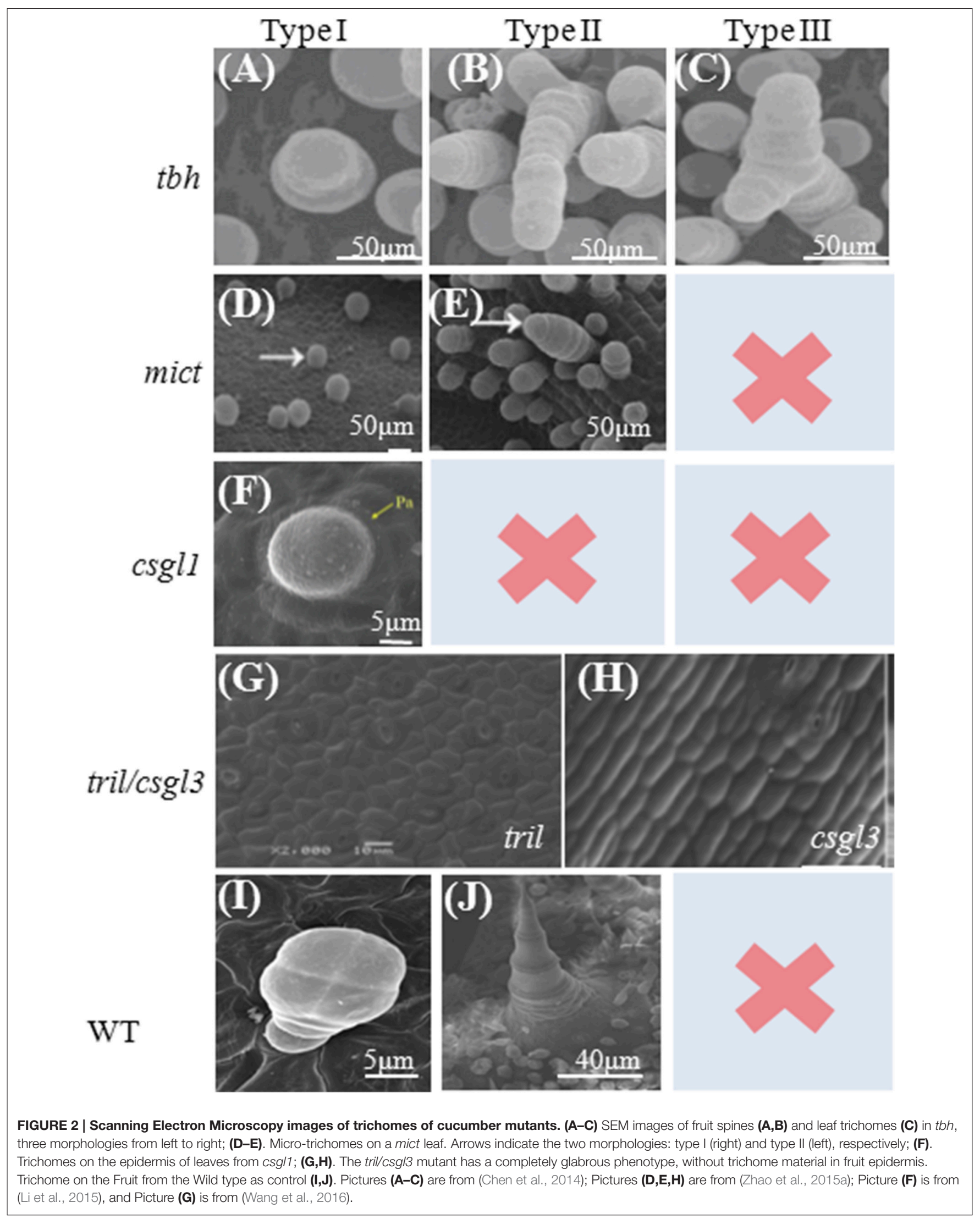


TABLE 1 | Previous morphological studies of trichome in tbh, csgl1, mict, tril and csgl3.

\begin{tabular}{llll}
\hline $\begin{array}{l}\text { Mutant } \\
\text { name }\end{array}$ & Origin & Types of trichome described & References \\
\hline tbh & Northern China & $\begin{array}{l}\text { Type I: tiny 3- to 5-cell base topped with a 4- to 8-cell head } \\
\text { Type II: non-glandular and branchless }\end{array}$ & Chen et al., 2014 \\
mict & Northern China & Type I: only a small papillar-shaped head Type II: 1-5 cells without the pyramid shaped head & Zhao et al., 2015a,b \\
csgl1 & Northern China & No trichomes on leave except glandular trichomes & Li et al., 2015 \\
tril & European greenhouse & No any types trichome & Wang et al., 2016 \\
csgl3 & European greenhouse & No any types trichome & Pan et al., 2015 \\
\hline
\end{tabular}

but none were found in csgll, suggesting trichome distribution is specific (Chen et al., 2014; Li et al., 2015; Zhao et al., 2015a).

\section{TBH, MICT, AND CSGL1 ARE THE ALLELIC WITH ALTERNATIVE SPLICING}

To decipher the molecular defects in the mict, csgll, and $t b h$ mutants, a map-based cloning approach was undertaken by three independent groups to isolate these genes. The results showed that TBH, MICT, and CsGL1 are allelic and that there was a $2649 \mathrm{bp}$ fragment deletion from -189 to $2460 \mathrm{bp}$ of the start codon in Csa3M748220 in the mict, csgll, and tbh mutants (Li et al., 2015; Zhao et al., 2015a) (Figure 3A). According to the gene ID that provided in the references, we extracted their proteins from the cucumber genome database (http://www.icugi.org/cgi-bin/ICuGI/genome/search.cgi) and analyzed their protein domains online (https://blast.ncbi.nlm. nih.gov/Blast.cgi). These genes were predicted to encode a class I homeodomain-leucine zipper (HD-Zip) protein consisting of different amino acid residues. For example, CsGL1 and TBH contain 240 amino acids with the conserved HD (65AA120AA) and Zip domains (121AA-164AA), but the MICT protein consists of 242 amino acid residues with a HALZ motif. Notably, there were two isoforms of this gene in the cucumber genome database (Csa3M748820.1 and Csa3M748820.2); therefore, we infer that the different protein lengths encoded by the same gene suggest that Csa3M748220 may exist by alternative splicing in cucumber. Subcellular localization showed that the Csa3M748220 coding sequence was fused to GFP (35S) in the nuclei of tobacco and onion epidermal cells (Zhao et al., 2015a,b). Moreover, a transcriptional activation activity assay in yeast found that Csa3M748220 had weak activity as a transcriptional activator (Zhao et al., 2015a). Therefore, based on the above results, Csa3M748220 has the typical features of a transcription factor. HD-Zip proteins are unique to the plant kingdom and can be classified into four groups, I-IV, based on their distinctive traits of DNA-binding specificities, gene structures, and common motifs (Abe et al., 2003; Hülskamp et al., 2005). HD-Zip I genes have been demonstrated to be involved largely in biological processes, such as abiotic stress responses, meristem regulation, and trichome development (Hanson et al., 2001; Himmelbach et al., 2002; Hjellström et al., 2003; Saddicl et al., 2006; Zhao et al., 2015a,b).

\section{TRIL, CSGL3 ARE THE ALLELIC WHICH OVERRIDE THE EFFECT OF TBH/MICT/ CSGL1 ON THE REGULATION OF MULTICELLULAR TRICHOME INITIATION AND DEVELOPMENT}

Thereare few reports on the regulatory genes that control multicellular trichome development in cucumber. This presents a good opportunity to use these mutants to analyze their regulatory mechanisms in trichomes of cucumber. As mentioned above, tril and csgl3 show a completely different glabrous morphology from that of the other three trichome-developmentrelated mutants, and only the epidermal cells, including stomata and encircling guard cells, were visible (Figure 2G). This indicates that TRIL and CsGL3 function in trichome cell fate determination. A transcriptome profiling analysis among wildtype, tril, tbh, mict, and csgll revealed that TBH, MICT, and CsGL1 were not expressed in the tril mutant (baseMean 0) but were highly expressed in the wild-type (baseMean 518.25) (Zhao et al., 2015a). TRIL was mapped to Cas6M514870, a member of the class IV HD-Zip family that shares $66.7 \%$ identity with PROTODERMAL FACTOR2 (PDF2, At4g04890.1), a shoot epidermal cell differentiation-related gene, and 35\% identity with GL2 (At1g79840.2), a gene that initiates trichome differentiation in Arabidopsis. In the tril mutant, there is a 5008 bp insertion fragment after the first exon (Figure 3B). CsGL3 was also mapped to Csa6M514870 by Pan and Cui (Pan et al., 2015; Cui et al., 2016). In Pan's study, the loss of function of the CsGL3 was due to the insertion of a 5-kb-long terminal repeat (LTR) retrotransposon in the 4 th exon of CsGL3 (Figure 3B). The insertion location is different from that of tril. Cui used three markers (InDel-19, dCAPs-2, and dCAPs-11) designed from the sequence of Csa6M514870, which co-segregated with the trait. In addition, Csa6M514870 was found to harbor 3 single-base substitutions: $\mathrm{T} \rightarrow \mathrm{C}(611 \mathrm{bp}), \mathrm{G} \rightarrow \mathrm{A}(820 \mathrm{bp})$, and $\mathrm{G} \rightarrow \mathrm{A}(865$ $\mathrm{bp}$ ) at the fourth exon, resulting in a change in the amino acid sequence. We still do not know how the CsGL3 changed in its corresponding mutant csgl3.

In the $t b h$, mict, and csgll mutants, TRIL/CsGL3 was overexpressed at different levels due to the different stages at which the samples were collected (Chen et al., 2014; Li et al., 2015; Zhao et al., 2015a). Moreover, the trichome phenotype analyzed in the F2 population between the tril and mict mutants suggests that the TRIL gene has a significant influence on trichome initiation and in determining the fate of epidermal cells, 


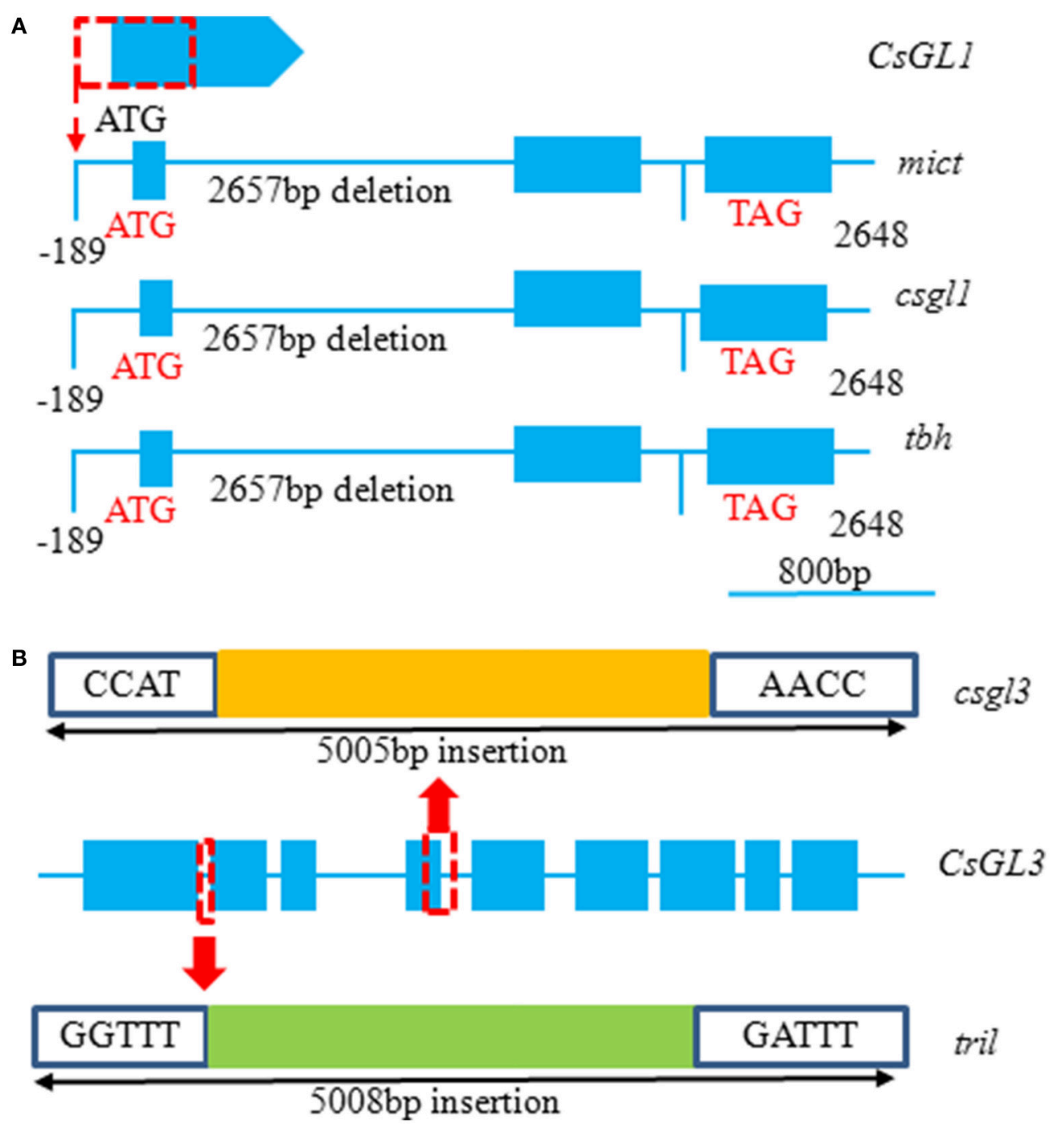

FIGURE 3 | Gene structure of CsGL1 and CsGL3 in the WT and mutants. (A) A 2649 bp deletion of the start codon in CsGL1 in the mict, csgl1 and tbh mutants compared to the WT. Boxes represent exons, and lines represent introns and intergenic regions. ATG and TAG indicate start and stop codons, respectively, and numbers represent nucleotide deletion length in mutants. Mict is from Zhao et al. (2015a); csgl1 is from Li et al. (2015). (B) A 5005 bp insertion at the 4th exon in csgl3 and a 5008 bp insertion after the first exon in the tril mutant. The red box indicates insertion sites, and yellow and green boxes represent insertion length. Different insertions in this gene showed the same phenotype in cucumber. tril is from Wang et al. (2016); csg/3 is from Pan et al. (2015).

whereas the MICT/TBH/CsGL1 gene only influences trichome development at the shoot. Genetically, based on the doublemutant phenotype in csgl3csgll, the TRIL/GsGL3 gene is assumed to act upstream of the MICT/TBH/CsGL1 gene and control the expression of MICT/TBH/ CsGL1 (Zhao et al., 2015a; Wang et al., 2016).

\section{MODELS FOR TRICHOME PATTERNING IN CUCUMBER MAY DIFFER FROM MODELS IN ARABIDOPSIS}

In the model plant Arabidopsis, the activator-inhibitor model has guided intuitive modeling and experimental design for a long time because it offers a reasonable explanation for the apparently paradoxical situation in that trichome-promoting and trichomeinhibiting genes are both expressed strongly in trichomes (Pesch and Hülskamp, 2009). Here, we focus on a positive regulatory model for trichome development between Arabidopsis and cucumber. The main reason is that all of the mutants in cucumber reveal fewer or no trichomes visually, suggesting that they encode a positive regulator of trichome development. Solid evidence of the genetic basis of trichome initiation has identified genes that (a) control the entry into the trichome pathway and (b) control the spacing of initiation events. Lossof-function mutations in Arabidopsis thaliana have suggested that the genes GL1 (which encodes an MYB transcription factor) and TTG1 (which encodes a WD40 repeat-containing protein) are important for the initiation and spacing of leaf trichomes (Galway et al., 1994; Walker et al., 1999), while for normal 
trichome initiation, the genes GL3, and EGL3 (Payne et al., 2000; Zhang et al., 2003), which encode helix-loop-helix (bHLH) proteins, are needed (Figure 4A).

In cucumber, the TRIL/CsGL3 gene encodes a HD-Zip IV protein. The loss-of-function mutant showed that tril/csgl3 has a trichome-less phenotype, indicating that this gene is a positive regulator of trichome development. Its homologous genes PDF2 and ARABIDOPSIS THALIANA MERISTEM LAYER1 (ATML1) in Arabidopsis also encode HD-Zip IV protein family members (Nakamura et al., 2006). The pdf2atml1 double mutant displays striking defects in shoot epidermal cells (Abe et al., 2003). In tomato, another typical multicellular trichome plant, Wo RNAi transgenic tomato plants showed similar shoot and root epidermal cell formation as that of the tril mutant. Wo shares an amino acid sequence identical to that of TRIL/CsGL3 and belongs to the same HD-Zip IV protein family. These results indicate that the role of TRIL/CsGL3 in the initiation and control of multicellular trichrome pathways. Based on previous results, a proposed model explaining trichome patterning in the positive regulator pathway in cucumber was built (Figure 4B). In this model, HD-Zip transcription factors may bind to other types of transcription factors to generate a specific complex to control cucumber multicellular trichome formation.

Based on transcriptional data from all of the mutants, several candidate genes should be focused on extensively (Chen et al., 2014; Zhao et al., 2015a,b). For example, the CsMYB6, CsWIN1, and CsGL2 genes were down-regulated not only in tril/csgl3 but also in tbh, mict, and $c s g l$, indicating that those genes were involved in multicellular trichome development in cucumber.

\section{DISCUSSIONS}

In the past decade, substantial progress has been made in delineating the genes that control trichome development in

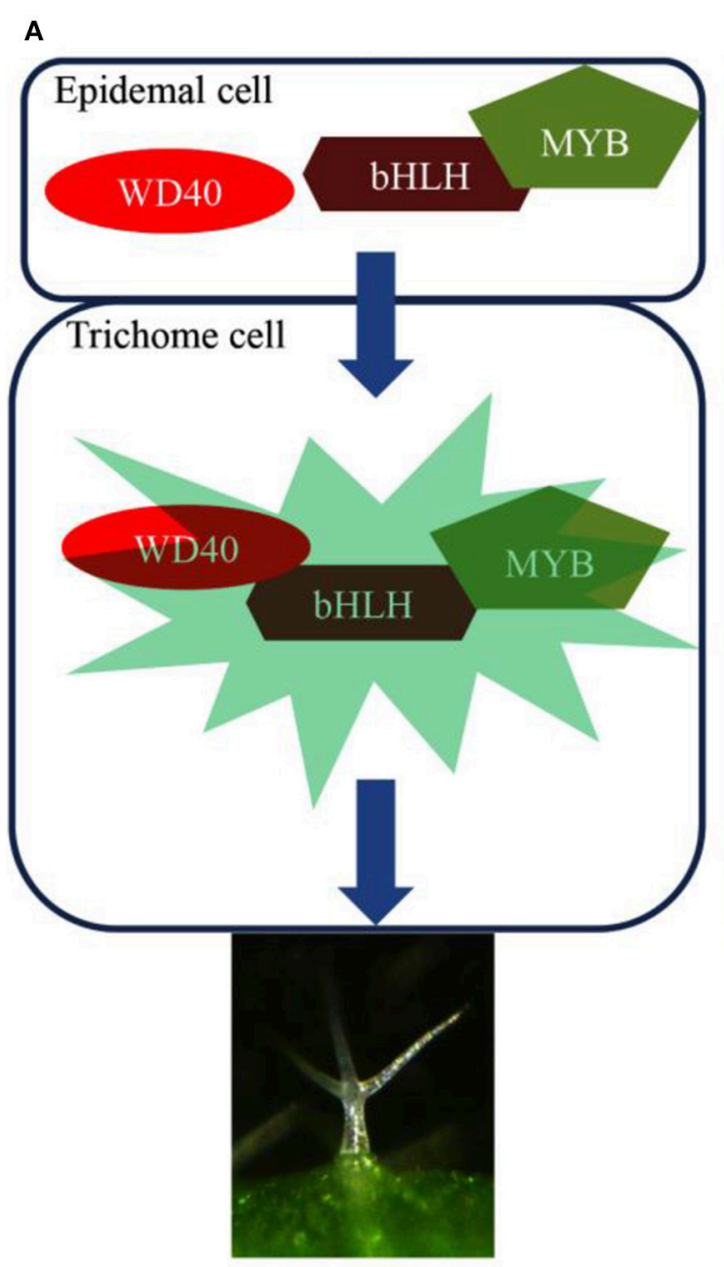

B

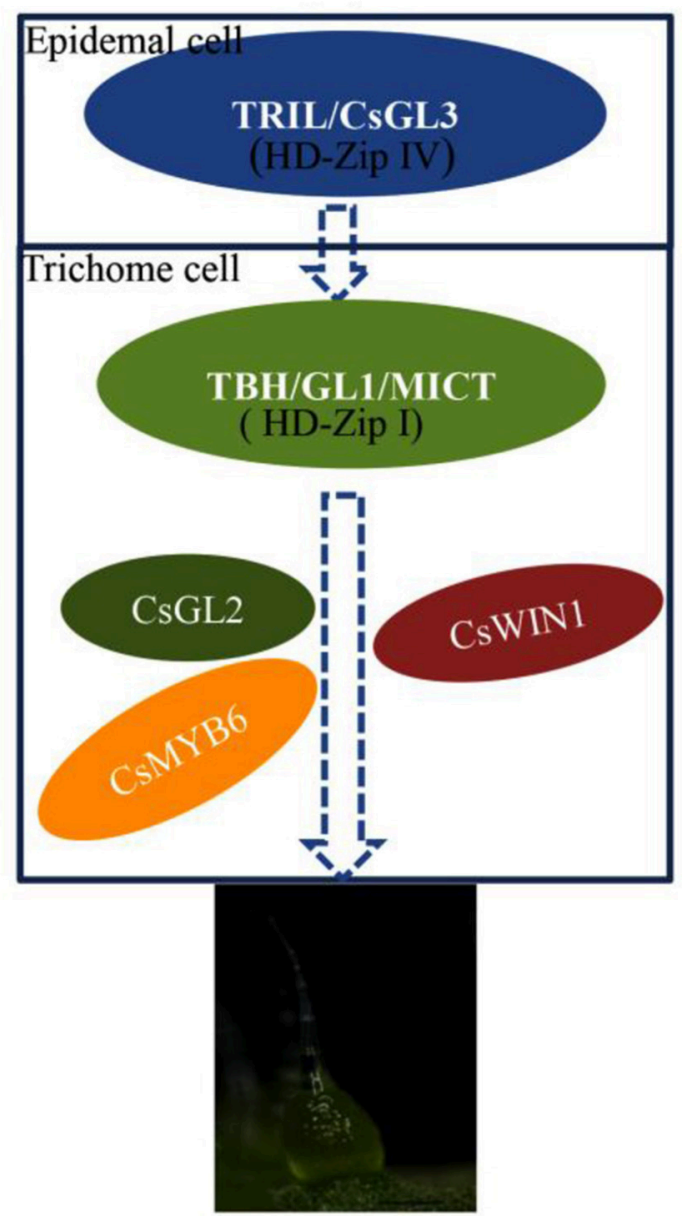

FIGURE 4 | Proposed model to explain trichome patterning in the positive regulator pathway in cucumber and in Arabidopsis. (A) TRIL/CsGL3 encodes the HD-Zip IV protein and may directly interact with the HD-Zip I protein (MICT/TBH/CsGL1) to regulate trichome development in cucumber. CsMYB6, CsWIN1, and CsGL2 may be located downstream of MICT/TBH/CsGL1 and positively participate in trichome development.(B) In unicellular trichomes, WD40, Bhlh, and MYB form a complex to positively regulate trichome development. The core complex in MBW (A) may differ from the HD-Zip complex (B). The bold arrows in the boxes indicate developmental progression. 
cucumber. Several groups provide evidence to suggest that a number of transcriptional activators, such as HD-Zip I and HD-Zip IV, play a role in fine tuning the spatial and temporal distribution of trichomes. Researchers have tried to demonstrate the possible mechanisms of these transcriptional activators.

However, much remains unknown and needs to be elucidated in future research. The functions of the HD-Zip IV and HD-Zip I genes require further investigation through genetic transformation in cucumber plants. Our current knowledge about the gene regulatory networks is largely limited to the unicellular trichomes in the model plant Arabidopsis. However, little is known about the regulatory network that controls the development of multicellular trichomes in cucumber. Evidence indicates that the common regulatory mechanisms of unicellular trichomes in Arabidopsis or of multicellular trichomes in cotton involve plant-specific genes that function distinctively. An analysis of the differential expression data generated by RNA-seq can offer new information for identifying putative key multicellular development transcription factors in cucumber. This is why we focused on the critical transcription factor genes. A new set of more than 42 transcription factor genes, including Homeodomain, MCMI-AGAMOUSDEFICIENS-SRF4 (MADS), and WRKY domains, has been identified in a transcriptome analysis of all cucumber trichomerelated mutants (Zhao et al., 2015a). These transcription factor genes are unique to plants and are involved in a range of activities; many are associated with multicellular trichrome development and other species-specific development processes. The study of the interaction among those transcription factors is an emerging area of research because these factors probably share many biological functions for trichome development. All of this information warrants further investigation.

The aim of this review is to provide readers with a summary of the progress of cucumber trichome development and to

\section{REFERENCES}

Abe, M., Katsumata, H., Komeda, Y., and Takahashi, T. (2003). Regulation of shoot epidermal cell differentiation by a pair of homeodomain proteins in Arabidopsis. Development 130, 635-643. doi: 10.1242/dev.00292

Balkunde, R., Pesch, M., and Hülskamp, M. (2010). Trichome patterning in Arabidopsis thaliana: from genetic to molecular models. Curr. Top. Dev. Biol. 91, 299-321. doi: 10.1016/S0070-2153(10)91010-7

Chen, C., Liu, M., Jiang, L., Liu, X., Zhao, J., Yan, S., et al. (2014). Transcriptome profiling reveals roles of meristem regulators and polarity genes during fruit trichome development in cucumber (Cucumis sativus L.). J. Exp. Bot. 65, 4943-4958. doi: 10.1093/jxb/eru258

Cui, J., Miao, H., Ding, L., Wehner, T. C., Liu, P., Wang, Y., et al. (2016). A new glabrous Gene ( $\operatorname{csgl} 3)$ identified in trichome development in cucumber (Cucumis sativus L.). PLoS ONE 11:e0148422. doi: 10.1371/journal.pone.0148422

Edgar, B. A., Zielke, N., and Gutierrez, C. (2014). Endocycles: a recurrent evolutionary innovation for post-mitotic cell growth. Nat. Rev. Mol. Cell Biol. 15, 197-210. doi: 10.1038/nrm3756

Esch, J. J., Chen, M., Hillestad, M., and David, M. M. (2004). Comparison of TRY and the closely related At1g01380 gene in controlling Arabidopsis trichome patterning. Plant J. 40, 860-869. doi: 10.1111/j.1365-313X.2004. 02259.x encourage plant scientists to further investigate the mechanism of trichrome initiation and development and their regulatory network. We have summarized the mutants related to fruit trichomes, the key genes that control trichome initiation and development, gene relationships and a possible model for fruit trichome positive regulatory mechanisms that is different from Arabidopsis in core transcriptional factor numbers. All of this may help us to better understand the advances in the study of cucumber trichomes. At the same time, more investigations must be conducted.

Because cucumber is a horticultural crop of worldwide importance and fruit spines directly affect its commercial quality, an extensive characterization of cucumber trichomes will not only help us to understand the underlying molecular mechanisms involved in multicellular trichome development but will also pave the way for creating new cucumber varieties with desired trichome growth and density. Moreover, cucumber trichomes may serve as a model system for studying the development of multicellular trichomes.

\section{AUTHOR CONTRIBUTIONS}

HR and XL organized the review, wrote the first draft and generated Figures 1-4. EB and YC contributed to a second draft. All of the authors revised the manuscript multiple times. HR and $\mathrm{XL}$ performed the final revision of the manuscript, which was read and approved by all authors.

\section{ACKNOWLEDGMENTS}

We thank the members of the Ren lab for helpful discussions for this review. This work was supported by National Research and Development Program (2016YFD0101705), Beijing Agricultural Innovation Consortium (BAIC01-2016) and Beijing Agricultural Scientific and Technological Program (20160415) to HR.
Galway, M. E., Masucci, J. D., Lloyd, A. M., Walbot, V., Davis, R. W., and Schiefelbein, J. W. (1994). The TTG gene is required to specify epidermal cell fate and cell patterning in the Arabidopsis root. Dev. Biol. 166, 740-754. doi: 10.1006/dbio.1994.1352

Grebe, M. (2012). The patterning of epidermal hairs in Arabidopsis-updated. Curr. Opin. Plant Biol. 15, 31-37. doi: 10.1016/j.pbi.2011.10.010

Hanson, J., Johannesson, H., and Engström, P. (2001). Sugar-dependent alterations in cotyledon and leaf development in transgenic plants expressing the HDZhdip gene ATHB13. Plant Mol. Biol. 45, 247-262. doi: 10.1023/A:1006464907710

Hauser, M. T. (2014). Molecular basis of natural variation and environmental control of trichome patterning. Front. Plant Sci. 5:320. doi: 10.3389/fpls.2014.00320

Himmelbach, A., Hoffmann, T., Leube, M., Höhener, B., and Grill, E. (2002) Homeodomain protein ATHB6 is a target of the protein phosphatase ABI1 and regulates hormone responses in Arabidopsis. EMBO J. 21, 3029-3038. doi: 10.1093/emboj/cdf316

Hjellström, M., Olsson, A. S., Engström, P., and Söderman, E. (2003). Constitutive expression of the water deficit-inducible homeobox gene ATHB7 in transgenic Arabidopsis causes a suppression of stem elongation growth. Plant Cell Environ. 26, 1127-1136. doi: 10.1046/j.1365-3040.2003.01037.x

Hülskamp, J. A., Walker, A. R., Timmis, J. N., and Orford, S. J. (2005). Two WDrepeat genes from cotton are functional homologues of the Arabidopsis thaliana 
TRANSPARENT TESTA GLABRA1 (TTG1) gene. Plant Mol. Biol. 57, 67-81. doi: 10.1007/s11103-004-6768-1

Hülskamp, M. (2004). Plant trichomes: a model for cell differentiation. Nat. Rev. Mol. Cell Biol. 5, 471-480. doi: 10.1038/nrm1404

Hülskamp, M., Schnittger, A., and Folkers, U. (1998). Pattern formation and cell differentiation: trichomes in Arabidopsis as a genetic model system. Int. Rev. Cytol. 186, 147-178. doi: 10.1016/S0074-7696(08)61053-0

Ishida, T., Kurata, T., Okada, K., and Wada, T. (2008). A genetic regulatory network in the development of trichomes and root hairs. Annu. Rev. Plant Biol. 59, 365-386. doi: 10.1146/annurev.arplant.59.032607.092949

Kirik, V., Simon, M., Wester, K., Schiefelbein, J., and Hulskamp, M. (2004). ENHANCER of TRYand CPC2 (ETC2) reveals redundancy in the regionspecific control of trichome development of Arabidopsis. Plant Mol. Biol. 55, 389-398. doi: 10.1007/s11103-004-0893-8

Li, Q., Cao, C., Zhang, C., Zheng, S., Wang, Z., Wang, L., et al. (2015). The identification of Cucumis sativus Glabrous 1 (CsGL1) required for the formation of trichomes uncovers a novel function for the homeodomainleucine zipper I gene. J. Exp. Bot. 66, 2515-2526. doi: 10.1093/jxb/erv046

Li, S., Milliken, O. N., Pham, H., Seyit, R., Napoli, R., Preston, J., et al. (2009). The Arabidopsis MYB5 transcription factor regulates mucilage synthesis, seed coat development, and trichome morphogenesis. Plant Cell 21, 72-89. doi: 10.1105/tpc.108.063503

Liu, X., Wang, L., Liu, L., Guo, Y., and Ren, H. (2011). Alleviating effect of exogenous nitric oxide in cucumber seedling against chilling stress. Afr. J. Biotechnol. 10, 4380-4386. doi: 10.5897/AJB10.812

Nakamura, M., Katsumata, H., Abe, M., Yabe, N., Komeda, Y., Yamamoto, K. T., et al. (2006). Characterization of the class IV homeodomain-leucine zipper gene family in Arabidopsis. Plant Physiol. 141, 1363-1375. doi: 10.1104/pp.106.077388

Oppenheimer, D. G., Herman, P. L., Sivakumaran, S., Esch, J., and Marks, M. D. (1991). A myb gene required for leaf trichome differentiation in Arabidopsis is expressed in stipules. Cell 67, 483-493. doi: 10.1016/0092-8674(91) 90523-2

Pan, Y., Bo, K., Cheng, Z., and Weng, Y. (2015). The loss-of-function GLABROUS 3 mutation in cucumber is due to LTR-retrotransposon insertion in a class IV HD-ZIP transcription factor gene CsGL3 that is epistatic over CsGL1. BMC Plant Biol. 15:302. doi: 10.1186/s12870-015-0693-0

Payne, C. T., Zhang, F., and Lloyd, A. M. (2000). GL3 encodes a bHLH protein that regulates trichome development in Arabidopsis through interaction with GL1 and TTG1. Genetics 156, 1349-1362.

Pesch, M., and Hülskamp, M. (2009). One, two, three...models for trichome patterning in Arabidopsis? Curr. Opin. Plant Biol. 12, 587-592. doi: 10.1016/j.pbi.2009.07.015

Saddicl, A., Huvermann, B., Bezhani, S., Su, Y., Winter, C. M., et al. (2006). The LEAFY target LMI1 is a meristem identity regulator and acts together with LEAFY to regulate expression of CAULIFLOWER. Development 133, 1673-1682. doi: $10.1242 /$ dev.02331

Schwab, B., Folkers, U., Ilgenfritz, H., and Hülskamp, M. (2000). Trichome morphogenesis in Arabidopsis. Philos Trans. Soc. Lond. B Biol. Sci. 355, 879-883. doi: 10.1098/rstb.2000.0623

Szymanski, D. B., Lloyd, A. M., and Marks, M. D. (2000). Progress in the molecular genetic analysis of trichome initiation and morphogenesis in Arabidopsis. Trends Plant Sci. 5, 214-219. doi: 10.1016/S1360-1385(00)01597-1

Tissier, A. (2012). Glandular trichomes: what comes after expressed sequence tags? Plant J. 70, 51-68. doi: 10.1111/j.1365-313X.2012.04913.x

Tominaga-Wada, R., Nukumizu, Y., Sato, S., Kato, T., Tabata, S., and Wada, T. (2012). Functional divergence of MYB-related genes, WEREWOLF and AtMYB23 in Arabidopsis. Biosci. Biotechnol. Biochem. 76, 883-887. doi: 10.1271/bbb.110811
Wada, T., Kurata, T., Tominaga, R., Koshino-Kimura, Y., Tachibana, T., Goto, K., et al. (2002). Role of a positive regulator of root hair development, CAPRICE, in Arabidopsis root epidermal cell differentiation. Development 129, 5409-5419. doi: $10.1242 / \mathrm{dev} .00111$

Wada, T., Tachibana, T., Shimura, Y., and Okada, K. (1997). Epidermal cell differentiation in Arabidopsis determined by a Myb homolog, CPC. Science 277, 1113-1116. doi: 10.1126/science.277.5329.1113

Walker, A. R., Davison, P. A., Bolognesi-Winfield, A. C., James, C. M., Srinivasan, N., Blundell, T. L., et al. (1999). The TRANSPARENT TESTA GLABRA1 locus, which regulates trichome differentiation and anthocyanin biosynthesis in Arabidopsis, encodes a WD40 repeat protein. Plant Cell 11, 1337-1349. doi: 10.1105/tpc.11.7.1337

Wang, S., and Chen, J. G. (2014). Regulation of cell fate determination by singlerepeat R3 MYB transcription factors in Arabidopsis. Front. Plant Sci. 5:133. doi: 10.3389/fpls.2014.00133

Wang, S., Wang, J. W., Yu, N., Li, C. H., Luo, B., et al. (2014). Control of plant trichome development by a cotton fiber MYB gene. Plant Cell 16, 2323-2334. doi: 10.1105/tpc.104.024844

Wang, Y., Nie, J., Chen, H., Guo, C., Pan, J., He, H., et al. (2016). Identification and mapping of Tril, a homeodomain-leucine zipper gene involved in multicellular trichome initiation in Cucumis sativus. Theor. Appl. Genet. 129, 305-316. doi: 10.1007/s00122-015-2628-4

Wester, K., Digiuni, S., Geier, F., Timmer, J., Fleck, C., and Hülskamp, M. (2009). Functional diversity of R3 single-repeat genes in trichome development. Development 136, 1487-1496. doi: 10.1242/dev.021733

Yang, X., Zhang, W., He, H., Nie, J., Bie, B., Zhao, J., et al. (2014). Tuberculate fruit gene Tu encodes a $\mathrm{C} 2 \mathrm{H} 2$ zinc finger protein that is required for the warty fruit phenotype in cucumber (Cucumis sativus L.). Plant J. 78, 1034-1046. doi: $10.1111 /$ tpj. 12531

Zhang, F., Gonzalez, A., Zhao, M., and Payne, C. T. (2003). Lloyd A. A network of redundant bHLH proteins functions in all TTG1-dependent pathways of Arabidopsis. Development 130, 4859-4869. doi: 10.1242/dev.00681

Zhang, W., He, H., Guan, Y., Du, H., Yuan, L., Li, Z., et al. (2010). Identification and mapping of molecular markers linked to the tuberculate fruit gene in the cucumber (Cucumis sativus L.). Theor. Appl. Genet. 120, 645-654. doi: 10.1007/s00122-009-1182-3

Zhao, H., Wang, X., Zhu, D., Cui, S., Li, X., Cao, Y., et al. (2012). A single amino acid substitution in IIIf subfamily of basic helix-loop-helix transcription factor AtMYC1 leads to trichome and root hair patterning defects by abolishing its interaction with partner proteins in Arabidopsis. J. Biol. Chem. 287, 14109-14121. doi: 10.1074/jbc.M111.280735

Zhao, J., Pan, J., Guan, Y., Nie, J., Yang, J., Qu, M., et al. (2015b). Transcriptome analysis in Cucumis sativus identifies genes involved in multicellular trichome development. Genomics 105, 296-303. doi: 10.1016/j.ygeno.2015.01.010

Zhao, J., Pan, J., Guan, Y., Zhang, W., Bie, B., Wang, Y., et al. (2015a). Microtrichome as a class I homeodomain-leucine zipper gene regulates multicellular trichome development in Cucumis sativus. J. Integr. Plant Biol. 57, 925-935. doi: 10.1111/jipb.12345

Conflict of Interest Statement: The authors declare that the research was conducted in the absence of any commercial or financial relationships that could be construed as a potential conflict of interest.

Copyright (c) 2016 Liu, Bartholomew, Cai and Ren. This is an open-access article distributed under the terms of the Creative Commons Attribution License (CC BY). The use, distribution or reproduction in other forums is permitted, provided the original author(s) or licensor are credited and that the original publication in this journal is cited, in accordance with accepted academic practice. No use, distribution or reproduction is permitted which does not comply with these terms. 